03

\title{
О связи $T$-матриц, возникающих в осесимметричной задаче рассеяния света сфероидом
}

\author{
(C) В.Г. Фарафоонов ${ }^{1}$, В.Б. Ильин ${ }^{1,2,3}$, Д.Г. Туричина ${ }^{2}$ \\ ${ }^{1}$ Государственный университет аэрокосмического приборостроения, \\ 190000 Санкт-Петербург, Россия \\ ${ }^{2}$ Санкт-Петербургский государственный университет, \\ 199034 Санкт-Петербург, Россия \\ ${ }^{3}$ Главная (Пулковская) астрономическая обсерватория РАН, \\ 196140 Санкт-Петербург, Россия \\ e-mail: far@aanet.ru
}

Поступила в редакцию 30.10.2021 г.

В окончательной редакции 30.10.2021 г.

Принята к публикации 12.11.2021 г.

Найдены соотношения между $T$-матрицами, возникающими при решении проблемы рассеяния света сфероидом с использованием разложений электромагнитных полей в применяемых сфероидальных и сферических базисах. Численно исследовано поведение найденных соотношений и отмечено, что в широкой области значений параметров задачи вычисление сфероидальной $T$-матрицы и соответствующее ее преобразование единственный практический способ получения сферической $T$-матрицы, часто используемой в приложениях.

Ключевые слова: рассеяние света, $T$-матрица.

DOI: $10.21883 /$ OS.2022.02.51995.2893-21

\section{Введение}

Представление реальных рассеивателей сфероидами является подходом, широко применяемым в разных областях науки: например, в оптике атмосферы [1-3], астрономии [4], медицине [5], нанооптике [6], лабораторном анализе [7] и т.д. Особенно часто используется сфероидальная модель в современной астрофизике из-за отсутствия информации о форме несферических космических пылинок [8-12].

Оптические свойства сфероидов могут быть рассчитаны разными методами. Наиболее естественно использовать метод разделения переменных в сфероидальных координатах, связанных с поверхностью частицы, т. е. раскладывать поля в соответствующем сфероидальном базисе $[13,14]$. За естественность приходится платить трудностью вычисления сфероидальных функций и сложностью уравнений. Более простой подход использовать разложение полей в сферическом базисе и далее метод $T$-матриц [15]. При этом для ансамбля сфероидов среднение по ориентациям можно делать аналитически. Недостатком подхода является то, что вычислительные проблемы для сфероидов быстро нарастают с ростом их дифракционного параметра, отношения полуосей или показателя преломления. В частности, в астрофизических приложениях оказывается критичным первое и иногда второе [16,17], при микроволновых экспериментах — третье [18]. Кроме перечисленных для расчета оптических свойств сфероидов можно применять универсальные методы, разработанные для частиц произвольной формы и строения (см., например, обзор в [19]). Однако область применимости этих методов заметно уже, чем области применимости методов разделения переменных и $T$-матриц [20], а кроме этого универсальные методы являются относительно медленными при вычислениях.

Широко известны две компьютерные программы для расчета рассеяния света сфероидами методом разделения переменных в сфероидальных координатах: код Asano \& Yamamoto [21] и код Вощинникова и Фарафонова [14]. В первом применялся стандартный базис ${ }^{1}$, во втором - оригинальный неортогональный, что было найдено более эффективным с вычислительной точки зрения [14]. Обе программы имеют возраст более 30 лет. За это время появились новые, более устойчивые алгоритмы вычисления сфероидальных функций [22-24] и стала понятна прикладная значимость сферической $T$-матрицы [25]. В связи с этим возникает вопрос о расчете сфероидальной $T$-матрицы и ее преобразовании в сферическую.

В настоящей статье мы рассматриваем осесимметричную задачу рассеяния света сфероидом, которая является репрезентативной частью полной задачи, и рассчитываем для нее $T$-матрицу при сфероидальном базисе, связанном с поверхностью частицы. Затем находим способ преобразования этой сфероидальной $T$-матрицы в сферическую, возникающую при использовании тех же скалярных потенциалов. Следующим шагом является обсуждение преобразования сферической $T$-матрицы,

\footnotetext{
${ }^{1}$ Базис, примененный в [21], является обобщением стандартного сферического базиса, возникающего при использовании потенциалов Дебая и лежащего в основе теории Ми.
} 
связанной с неортогональным сферическим базисом, в $T$-матрицу, определенную для стандартного ортогонального сферического базиса. Найденные соотношение между $T$-матрицами использованы для численных расчетов сечений рассеяния и ослабления для различных сфероидов, и на их основании проведено обсуждение полученных в работе результатов.

\section{1. Осесимметричная задача рассеяния света и ее решение}

\section{1. Основные уравнения}

Распространение электромагнитных волн описывается уравнениями Максвелла. В случае рассеяния света частицей обычно рассматриваются гармонические поля $\mathbf{E}(\mathbf{r}, \omega), \mathbf{H}(\mathbf{r}, \omega)$, которые зависят от положения точки $(\mathbf{r})$ и частоты излучения $(\omega)$ и удовлетворяют векторному уравнению Гельмгольца [26]:

$$
\Delta \mathbf{E}+k^{2} \mathbf{E}=0
$$

где $k-$ волновое число в среде, и не противоречат условию поперечности поля: $\nabla \cdot \mathbf{E}=0$.

Граничные условия в задаче рассеяния света задают непрерывность тангенциальных составляющих полей на поверхности частицы $S$ [26]:

$$
\begin{aligned}
\left(\mathbf{E}^{\text {in }}+\mathbf{E}^{\text {sca }}\right) \times \mathbf{n} & =\mathbf{E}^{\text {int }} \times \mathbf{n}, \quad\left(\mathbf{H}^{\text {in }}+\mathbf{H}^{\text {sca }}\right) \times \mathbf{n} \\
& =\mathbf{H}^{\text {int }} \times \mathbf{n}, \mathbf{r} \in S,
\end{aligned}
$$

где $\mathbf{E}^{\text {in }}, \mathbf{H}^{\text {in }}$ означают известное поле излучения, падающего на частицу, $\mathbf{E}^{\text {sca }}, \mathbf{H}^{\text {sca }}$ и $\mathbf{E}^{\text {int }}, \mathbf{H}^{\text {int }}-$ неизвестные поля соответственно рассеянного излучения и излучения внутри частицы, $\mathbf{n}$ - внешняя нормаль к $S$.

Приведенная выше дифференциальная формулировка задачи эквивалентна интегральной, основанной на формулах Стрэттона-Чу и включающей граничные условия [27]

$$
\begin{aligned}
& \nabla \times \int_{S} \mathbf{n} \times \mathbf{E}^{\mathrm{int}}\left(\mathbf{r}^{\prime}\right) G\left(\mathbf{r}, \mathbf{r}^{\prime}\right) \mathrm{d} s^{\prime} \\
& -\frac{1}{i k \varepsilon} \nabla \times \nabla \times \int_{S} \mathbf{n} \times \mathbf{H}^{\mathrm{int}}\left(\mathbf{r}^{\prime}\right) G\left(\mathbf{r}, \mathbf{r}^{\prime}\right) \mathrm{d} s^{\prime} \\
& = \begin{cases}-\mathbf{E}^{\text {in }}(\mathbf{r}), & \mathbf{r} \in D, \\
\mathbf{E}^{\mathrm{sca}}(\mathbf{r}), & \mathbf{r} \in R^{3} \backslash \bar{D}\end{cases}
\end{aligned}
$$

где $D-$ объем, занимаемый частицей, $G\left(\mathbf{r}, \mathbf{r}^{\prime}\right)=\exp \left(i k\left|\mathbf{r}-\mathbf{r}^{\prime}\right|\right) /\left(4 \pi\left|\mathbf{r}-\mathbf{r}^{\prime}\right|\right)-$ функция Грина, $\varepsilon$ - диэлектрическая проницаемость.

Мы будем использовать интегральную формулировку задачи и рассматривать в основном осесимметричные поля, т.е. поля, не зависящие от азимутального угла. Такие поля возникают, например, тогда, когда падающее излучение является излучением диполя, момент которого ориентирован параллельно оси симметрии частицы. Кроме этого, осесимметричные поля появляются при разложении полей по сферическим или сфероидальным гармоникам как компоненты с азимутальным числом $m$, равным 0. Проблема рассеяния света осесимметричными частицами сводится для таких компонентов к скалярной задаче, которая часто выделяется в отдельную подзадачу [14]. Последнее удобно для численного и теоретического анализа, поскольку, несмотря на относительную простоту, данная подзадача содержит все особенности решаемой задачи [28].

\section{2. Метод решения и $\boldsymbol{T}$-матрица}

Используем подход к решению проблемы рассеяния света, основанный на разложении полей по векторному базису и определении неизвестных коэффициентов разложения путем подстановки разложений в граничные условия (см. подробнее [27]).

Как известно, уравнение Гельмгольца (1) имеет следующие решения, подходящие для представления поперечных электромагнитных полей [26]:

$$
\mathbf{M}^{\mathbf{a}}=\nabla \times(\Psi \mathbf{a}), \quad \mathbf{N}^{\mathbf{a}}=\frac{1}{k} \nabla \times \mathbf{M}^{\mathbf{a}}=\frac{1}{k} \nabla \times \nabla \times(\Psi \mathbf{a}),
$$

где а может быть постоянным вектором, например ортом декартовой системы координат $\mathbf{i}_{z}$, или радиусомвектором $\mathbf{r}$, а функция $\Psi$ является решением соответствующего скалярного уравнения Гельмгольца. Будем использовать как сферические, так и сфероидальные координаты (и соответственно функции).

В сферической системе $(r, \theta, \varphi)$ решения скалярного уравнения Гельмгольца имеют вид $(i=1,3)$

$$
\Psi_{m n}^{(i)}(r, \theta, \varphi)=\frac{1}{\sqrt{2 \pi}} z_{n}^{(i)}(k r) \bar{P}_{n}^{m}(\cos \theta) \mathrm{e}^{i m \varphi},
$$

где $z_{n}^{(1)}(k r)$ - сферические функции Бесселя $j_{n}(k r)$, а $z_{n}^{(3)}(k r)$ - сферические функции Ганкеля 1-го рода $h_{n}^{(1)}(k r), \bar{P}_{n}^{m}(\cos \theta)$ - нормированные присоединенные функции Лежандра с нормировочным множителем $\tilde{N}_{n m}=[2(n+m) ! /(2 n+1) /(n-m) !]^{1 / 2}$.

В сфероидальной системе $(\xi, \eta, \varphi)$

$$
\Psi_{m n}^{(i)}(\xi, \eta, \varphi)=\frac{1}{\sqrt{2 \pi}} R_{m n}^{(i)}(c, \xi) \bar{S}_{m n}(c, \eta) \mathrm{e}^{i m \varphi},
$$

где $R_{m n}^{(i)}(c, \xi)$ - радиальные сфероидальные функции $i$ го рода $(i=1,3), \bar{S}_{m n}(c, \eta)$ - нормированные угловые сфероидальные функции с нормировочным множителем $N_{m n}(c)$ (см. подробнее [29]), $N_{m n}(0)=\tilde{N}_{m n}, c=k d / 2$ и $c=-i k d / 2$ для вытянутых и сплюснутых сферидальных координат соответствнно, $d-$ фокусное расстояние (см. подробнее, например, [30]).

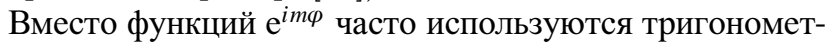
рические, и наряду с $m n$ вводится третий индекс $\sigma$, 
равный $e$ или $o$ в зависимости от того, используется $\cos m \varphi$ или $\sin m \varphi^{2}$. В результате, например, для базиса, включающего функции $\mathbf{M}^{\mathbf{a}}$ и $\mathbf{N}^{\mathbf{a}}$, разложение поля имеет вид

$$
\mathbf{E}=\sum_{m=0}^{\infty} \sum_{n=m}^{\infty}\left(a_{\sigma m n} \mathbf{M}_{\sigma m n}^{\mathbf{a}}+b_{\sigma m n} \mathbf{N}_{\sigma m n}^{\mathbf{a}}\right) .
$$

Поскольку задача рассеяния света является линейной, при таком подходе к ее решению естественным образом появляется так называемая $T$-матрица - матрица, связывающая векторы, содержащие коэффициенты разложения рассеянного излучения $a_{\sigma m n}^{\mathrm{sca}}, b_{\sigma m n}^{\mathrm{sca}}$ и падающего излучения $a_{\sigma m n}^{\text {in }}, b_{\sigma m n}^{\text {in }}$,

$$
\mathbf{a}^{\text {sca }}=T \mathbf{a}^{\text {in }} .
$$

Матрица $T$ содержит полную информацию об изменении излучения при рассеянии и полезна при рассмотрении хаотически ориентированных частиц, поскольку дает возможность аналитического усреднения сечений по ориентациям частицы [19].

\section{3. Скалярные потенциалы и их разложение в ряд}

В рассматриваемом подходе к решению задачи рассеяния света вместо полей часто используют их скалярные потенциалы. Существует несколько способов выбора скалярных потенциалов, каждый из которых имеет свои достоинства.

Широко известны потенцилы Дебая $\left(V_{\mathrm{e}}, V_{\mathrm{m}}\right)$. Они используются при рассмотрении рассеяния света шаром (теории Ми [31]) и сфероидом [21,32] и вводятся следующим соотношением, например, для ТЕ-моды:

$$
\mathbf{E}=i k_{0} \mu \nabla \times\left(V_{\mathrm{m}} \mathbf{r}\right)+\nabla \times \nabla \times\left(V_{\mathrm{e}} \mathbf{r}\right),
$$

где $k_{0}-$ волновое число в вакууме, $\mu-$ магнитная восприимчивость среды. Разложение этих потенциалов в ряд по решениям скалярного уравнения Гельмгольца $\Psi_{\text {бтn }}$ имеет вид

$$
V_{\mathrm{m}}=\sum_{m=0}^{\infty} \sum_{n=m}^{\infty} a_{\sigma m n} \Psi_{\sigma m n}, \quad V_{\mathrm{e}}=\sum_{m=0}^{\infty} \sum_{n=m}^{\infty} b_{\sigma m n} \Psi_{\sigma m n} .
$$

Важно отметить, что предствление (9) с разложениями (10) эквивалентны разложению поля (7) по векторному базису $\mathbf{M}^{\mathbf{r}}, \mathbf{N}^{\mathbf{r}}$ с теми же коэффициентами разложения $a_{\sigma m n}, b_{\sigma m n}$. Данное замечание относится и к другим потенциалам, рассмотриваемым ниже.

Иной набор потенциалов был использован в работе [14], где применялись потенциал Дебая $V$ и zкомпонент вектора Герца $U$. В этом случае для ТЕ-моды имеем

$$
\mathbf{E}=\nabla \times\left(U_{\mathrm{m}} \mathbf{i}_{z}\right)+\nabla \times\left(V_{\mathrm{m}} \mathbf{r}\right) .
$$

\footnotetext{
2 Тогда в соотношениях, аналогичных формулам (5), (6), появляется множитель $\sqrt{2-\delta_{m 0}}$, где $\delta_{m 0}-$ символ Кронекера [14].
}

Разложению этих потенциалов по функциям $\Psi_{\sigma m n}$ соответствует разложение полей по неортогональному базису $\mathbf{M}^{\mathbf{z}}, \mathbf{M}^{\mathbf{r}}$. Доказано, что таким образом достигаются заметные преимущества ${ }^{3}$ при рассмотрении рассеяния света сфероидами [14].

Кроме этого, для осесимметричных полей также использовались оригинальные потенциалы $p, q$, определенные следующим образом [14,33]:

$$
p=E_{\varphi} \cos \varphi, \quad q=H_{\varphi} \cos \varphi,
$$

где $\varphi-$ азимутальный угол, $E_{\varphi}$ и $H_{\varphi}-\varphi$-компоненты полей. Поскольку при осесимметричности полей $\mathbf{E}, \mathbf{H}$ не зависят от $\varphi$, а зависимость $p, q$ от $\varphi$ задается явным образом как косинус, то разложения потенциалов имеют вид

$$
p=\sum_{n=1}^{\infty} a_{n} \Psi_{e 1 n}, \quad q=\sum_{n=1}^{\infty} b_{n} \Psi_{e 1 n} .
$$

Таким образом, очевидно, что как разные системы координат, так и разные потенциалы приводят к различным векторным базисным функциям, используемым при разложении полей, и соответственно к разным коэффициентам их разложений и $T$-матрицам. Поэтому встает вопрос о связи таких матриц, который мы рассмотрим далее, начав с определения $T$-матрицы в случае осесимметричных полей, сфероидальных координат и потенциалов $p, q$.

\section{4. Решение осесимметричной задачи}

Будем решать задачу в сфероидальных координатах, следуя по возможности работе [34]. Подставим потенциалы полей $p$ в интегральные уравнения (3) и после некоторых простых преобразований получим соотношения

$$
\begin{aligned}
\int_{S}\left\{p^{\text {int }}\left(\mathbf{r}^{\prime}\right) \frac{\partial G\left(\mathbf{r}, \mathbf{r}^{\prime}\right)}{\partial n^{\prime}}-G\left(\mathbf{r}, \mathbf{r}^{\prime}\right) \frac{\partial}{\partial n^{\prime}} p^{\text {int }}\left(\mathbf{r}^{\prime}\right)\right\} \mathrm{d} s^{\prime} \\
= \begin{cases}-p^{\text {in }}(\mathbf{r}), & \mathbf{r} \in D, \\
p^{\mathrm{sca}}(\mathbf{r}), & \mathbf{r} \in R^{3} \backslash \bar{D} .\end{cases}
\end{aligned}
$$

Сначала будем решать скалярное интегральное уравнение для потенциала поля внутреннего излучения $p^{\text {int }}$ в области $D$, а затем потенциал поля рассеянного излучения $p^{\text {sca }}$ определим по известному потенциалу $p^{\text {int }}$. Решение уравнений для потенциала $q$ будет аналогичным [27].

Представим в уравнении (14) все потенциалы в виде разложений (13). Для потенциалов рассеянного поля используем коэффициенты $a_{l}^{\text {sca }}, b_{l}^{\text {sca }}$ и функции $\Psi_{e 1 n}^{(3)}\left(c_{1}, \mathbf{r}\right)$, для внутреннего поля $-a_{l}^{\text {int }}, b_{l}^{\text {int }}$ и $\Psi_{e 1 n}^{(1)}\left(c_{2}, \mathbf{r}\right)$ и для падающего излучения $-a_{l}^{\text {in }}, b_{l}^{\text {in }}$ и $\Psi_{e 1 n}^{(1)}\left(c_{1}, \mathbf{r}\right)$, где

\footnotetext{
${ }^{3}$ Связано с тем, что комбинируются функции, используемые для представления полей в проблеме рассеяния света шаром $\left(\mathbf{M}^{\mathbf{r}}\right)$ и бесконечным цилиндром $\left(\mathbf{M}^{\mathbf{Z}}\right)$.
} 
$c_{i}=k_{i} d / 2$ и $k_{1}$ и $k_{2}$ - волновые числа вне и внутри частицы соответственно. В случае плоской волны ТЕтипа коэффициенты известны [27]:

$$
a_{l}^{\text {in }}=-2 i^{l} \bar{S}_{1 l}\left(c_{1}, \cos \alpha\right), b_{l}^{\text {in }}=0,
$$

где $\alpha$ - угол между направлением распространения падающей волны и осью симметрии сфероида. Из соотношений (15) следует, что для такой волны отличен от нуля потенциал $p$. Для волны ТМ-типа имеем [27]

$$
a_{l}^{\text {in }}=0, \quad b_{l}^{\text {in }}=2 i^{l} \bar{S}_{1 l}\left(c_{1}, \cos \alpha\right),
$$

и ненулевым является только потенциал $q$.

Алгебраизация интегральных уравнений (14) после подстановки в них разложений потенциалов $p$ и функции Грина (ее разложение по сфероидальным функциям можно найти в монографии [29]) приводит к 2 бесконечным системам линейных уравнений относительно неизвестных коэффициентов разложений:

$$
\left\{\begin{array}{l}
\mathbf{z}^{\text {in }}=-A_{31} \mathbf{z}^{\text {int }}, \\
\mathbf{z}^{\text {sca }}=A_{11} \mathbf{z}^{\text {int }},
\end{array}\right.
$$

где $\quad$ векторы $\quad \mathbf{z}^{\text {in }}=\left\{z_{l}^{\text {in }}\right\}_{l=1}^{\infty}, \quad \mathbf{z}^{\text {sca }}=\left\{z_{l}^{\text {sca }}\right\}_{l=1}^{\infty}$, $\mathbf{z}^{\text {int }}=\left\{z_{l}^{\text {int }}\right\}_{l=1}^{\infty}$ имеют компоненты

$$
\begin{gathered}
z_{l}^{\text {in }}=a_{l}^{\text {in }} R_{1 l}^{(1)}\left(c_{1}, \xi_{0}\right), z_{l}^{\text {sca }}=a_{l}^{\text {sca }} R_{1 l}^{(3)}\left(c_{1}, \xi_{0}\right), \\
z_{l}^{\text {int }}=a_{l}^{\text {int }} R_{1 l}^{(1)}\left(c_{2}, \xi_{0}\right),
\end{gathered}
$$

диагональные матрицы равны

$$
\begin{gathered}
R_{i, j}=\left\{R_{1 l}^{(i) \prime}\left(c_{j}, \xi_{0}\right) / R_{1 l}^{(i)}\left(c_{j}, \xi_{0}\right) \delta_{n l}\right\}_{1}^{\infty}, \\
W_{1}=-\left[R_{3,1}-R_{1,1}\right]^{-1} \\
=\left\{i c_{1}\left(\xi_{0}^{2}-f\right) R_{1 l}^{(1)}\left(c_{1}, \xi_{0}\right) R_{1 l}^{(3)}\left(c_{1}, \xi_{0}\right) \delta_{n l}\right\}_{1}^{\infty}
\end{gathered}
$$

и, наконец,

$$
\begin{aligned}
& A_{31}=W_{1}\left\{R_{3,1} \Delta_{1,2}^{(1)}-\frac{\mu_{1}}{\mu_{2}} \Delta_{1,2}^{(1)} R_{1,2}-\left(\frac{\mu_{1}}{\mu_{2}}-1\right) \frac{\xi_{0}}{\xi_{0}^{2}-f} \Delta_{1,2}^{(1)}\right\}, \\
& A_{11}=W_{1}\left\{R_{1,1} \Delta_{1,2}^{(1)}-\frac{\mu_{1}}{\mu_{2}} \Delta_{1,2}^{(1)} R_{1,2}-\left(\frac{\mu_{1}}{\mu_{2}}-1\right) \frac{\xi_{0}}{\xi_{0}^{2}-f} \Delta_{1,2}^{(1)}\right\} .
\end{aligned}
$$

Здесь $\xi_{0}-$ значение координаты $\xi$ на поверхности сфероида, $f$ равно 1 для вытянутых частиц и -1 для сплюснутых. Элементы матриц $\Delta_{i, j}^{(1)}=\left\{\delta_{n l}^{(1)}\left(c_{i}, c_{j}\right)\right\}_{n, l=0}^{\infty}$ являются интегралами от произведения угловых сфероидальных функций и могут быть представлены в виде рядов

$$
\begin{aligned}
& \delta_{n l}^{(m)}\left(c_{i}, c_{j}\right)=\int_{-1}^{1} \bar{S}_{m n}\left(c_{i}, \eta\right) \bar{S}_{m l}\left(c_{j}, \eta\right) \mathrm{d} \eta= \\
& N_{m n}^{-1}\left(c_{i}\right) N_{m l}^{-1}\left(c_{j}\right) \sum_{r=0,1}^{\infty} d_{r}^{m n}\left(c_{i}\right) d_{r}^{m l}\left(c_{j}\right) \frac{2}{2 r+2 m+1} \frac{(r+m) !}{r !},
\end{aligned}
$$

где $d_{r}^{m n}-$ коэффиценты разложения угловой сфероидальной функции по присоединенным функциям Лежандра [29]. При одинаковых параметрах $\left(c_{i}=c_{j}\right)$ матрица $\Delta_{i j}^{(m)}$ совпадает с единичной.

Из уравнений (17) следует, что $\mathbf{z}^{\text {sca }}=-A_{11} A_{31}^{-1} \mathbf{z}^{\text {in }}$, и в результате получаем так называемую „сфероидальную“ (поскольку используются разложения по сфероидальным функциям) $T$-матрицу для потенциала $p$ :

$$
T_{p}^{s p}=-R_{3}^{-1} A_{11} A_{31}^{-1} R_{1},
$$

где $R_{i}=\left\{R_{1 n}^{(i)}\left(c_{1}, \xi_{0}\right) \delta_{n l}\right\}_{n, l=m}^{\infty}$ - диагональные матрицы $(i=1,3)$.

В численных расчетах будут использованы сечения ослабления $C_{\text {ext }}$ и рассеяния $C_{\text {sca }}$ излучения, которые, например, для ТЕ-моды равны [14]

$$
\begin{gathered}
C_{\text {ext }}=\frac{4 \pi}{k_{1}^{2}} \operatorname{Re} \sum_{l=1}^{\infty} i^{-l} a_{l}^{\text {sca }} \bar{S}_{1 l}\left(c_{1}, \cos \alpha\right), \\
C_{\text {sca }}=\frac{2 \pi}{k_{1}^{2}} \sum_{l=1}^{\infty}\left|a_{l}^{\text {sca }}\right|^{2} .
\end{gathered}
$$

В случае ТМ-моды соответствующие уравнения для потенциалов $q$ получаются из вышеприведенных после замены $\mu_{j} \rightarrow \varepsilon_{j}, \varepsilon_{j} \rightarrow \mu_{j}$ и $a_{n} \rightarrow b_{n}$. Аналогичные соотношения имеют место для сплюснутого сфероида и соответствующей системы координат.

\section{2. Преобразование $T$-матриц}

Естественно решать задачу рассеяния света сфероидом в сфероидальной системе, связанной с частицей и, следовательно, получать сфероидальную $T$-матрицу. Однако рядом полезных свойств обладают все-таки $T$ матрицы, определенные в сферической системе [19]. Поэтому сначала рассмотрим переход от разложений по сфероидальным функциям к разложениям по сферическим. Затем покажем, как сферические $T$-матрицы изменяются при смене базиса.

\section{1. Переход от сфероидальной к сферической $T$-матрице}

Соотношения между сфероидальными и сферическими функциями, удобные для решения этой задачи, были обобщены в работе [35]. Они выглядят следующим образом $(j=1,3)$ :

$$
\begin{aligned}
& R_{m n}^{(j)}(c, \xi) \bar{S}_{n m}(c, \eta)= \\
& =\sum_{l=m}^{\infty} i^{l-n} \frac{N_{m l}(0)}{N_{m n}(c)} d_{l-m}^{m n}(c) z_{l}^{(j)}(k r) \bar{P}_{l}^{m}(\cos \theta), \\
& z_{n}^{(j)}(k r) \bar{P}_{n}^{m}(\cos \theta) \\
& =\sum_{l=m}^{\infty} i^{n-l} \frac{N_{m n}(0)}{N_{m l}(c)} d_{n-m}^{m l}(c) R_{m l}^{(j)}(c, \xi) \bar{S}_{l m}(c, \eta) .
\end{aligned}
$$




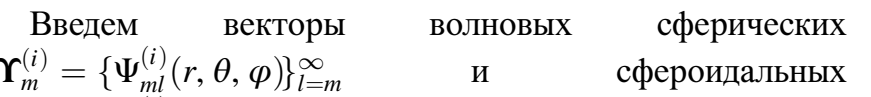
$\boldsymbol{\Psi}_{m}^{(i)}=\left\{\Psi_{m l}^{(i)}(\xi, \eta, \varphi)\right\}_{l=m}^{\infty}$ функций. Из соотношений (24) и (25) следует, что они связаны матричными соотношениями

$$
\begin{gathered}
\boldsymbol{\Psi}_{m}^{(i)}=D_{m}(c) \mathbf{\Upsilon}_{m}^{(i)}, \\
\boldsymbol{\Upsilon}_{m}^{(i)}=D_{m}^{-1}(c) \mathbf{\Psi}_{m}^{(i)}=D_{m}^{T}(c) \boldsymbol{\Psi}_{m}^{(i)},
\end{gathered}
$$

где использована матрица $D_{m}(c)=\left\{D_{n l}^{m}(c)\right\}_{n, l=m}^{\infty}=$ $\left\{i^{l-n} d_{l-m}^{m n}(c) N_{m l}(0) / N_{m n}(c)\right\}_{n, l=m}^{\infty}$, а $T$ означает транспонирование.

Из равенства скалярных потенциалов в сфероидальной и сферической системах координат и свойств скалярных произведений находим связь между $T$ матрицами, полученными в сфероидальном $\left(T^{\mathrm{sp}}\right)$ и сферическом $\left(T^{\mathrm{s}}\right)$ базисах:

$$
T^{\mathrm{s}}=D_{m}(c) T^{\mathrm{sp}} D_{m}^{T}(c) .
$$

Найденное соотношение не зависит от вида скалярных потенциалов и, следовательно, применимо к любым из них, включая все рассмотренные в разд. 1.3.

Заметим, что ранее перевод сфероидальной $T$ матрицы в сферическую был рассмотрен лишь в работе [36], где использовался иной, существенно более громоздкий подход.

\section{2. Переход от потенциалов $p, q$ к потенциалам Дебая}

Осесимметричную задачу для сфероидальной частицы целесообразно решать, используя соответствующую сфероидальную систему и привлекая потенциалы $p, q[14]$. Получаемая в таком случае $T$-матрица описана в разд. 1.4 , а ее перевод на случай соответствующего сферического базиса — в разд. 2.1. Определим, как вычисленная в результате такого перевода $T$-матрица $\left(T_{p, q}^{\mathrm{s}}\right)$ связана со сферической $T$-матрицей, получаемой для стандартных потенциалов Дебая $V_{\mathrm{e}}, V_{\mathrm{m}}\left(T_{V, V}^{\mathrm{s}}\right)$.

Рассмотрим падающее излучение. Для осесимметричной части ТЕ-моды плоской волны имеем разложение потенциала $p$ по формуле (13) со следующими коэффициентами разложения ${ }^{4}$ и функциями:

$$
\begin{gathered}
a_{n}^{\text {in }}=-2 i^{n} \bar{P}_{n}^{1}(\cos \alpha), \\
\Psi_{e 1 n}^{(1)}=\frac{1}{\sqrt{\pi}} j_{n}(k r) \bar{P}_{n}^{1}(\cos \theta) \cos \varphi .
\end{gathered}
$$

С другой стороны, осесимметричное поле $\mathbf{E}_{\mathrm{AS}}$ имеет разложение (7), где для потенциалов Дебая должны быть применены функции $\mathbf{M}_{\sigma m n}^{\mathbf{r}}, \mathbf{N}_{\sigma m n}^{\mathbf{r}}$. При этом из-за осесимметричности поля все функции с $m \neq 0$ не нужны, а кроме того, очевидно, что $\mathbf{N}_{\sigma 0 n}^{\mathrm{r}}=0$ и $a_{e 00}=0$ [32]. Будем рассматривать азимутальный компонент поля

$$
E_{\mathrm{AS}, \varphi}=\sum_{n=1}^{\infty} f_{e 0 n}^{\mathrm{in}} M_{e 0 n, \varphi}^{\mathrm{r}},
$$

\footnotetext{
${ }^{4}$ Например, по аналогии с формулой (15).
}

где, согласно [32] и без учета нормировки функций Лежандра,

$$
\begin{gathered}
f_{e 0 n}^{\mathrm{in}}=-i^{n} \frac{(2 n+1)}{n(n+1)} \frac{\mathrm{d} P_{n}(\cos \alpha)}{\mathrm{d} \alpha}, \\
M_{e 0 n, \varphi}^{\mathrm{r}}=-\frac{1}{\sqrt{2 \pi}} j_{n}(k r) \frac{\mathrm{d} P_{n}(\cos \theta)}{\mathrm{d} \theta} .
\end{gathered}
$$

Используя известное соотношение

$$
P_{n}^{m}(x)=(-1)^{m}\left(1-x^{2}\right)^{\frac{m}{2}} \frac{\mathrm{d}^{m} P_{n}(x)}{\mathrm{d} x^{m}},
$$

перейдем к нормированным функциям Лежандра $\bar{P}_{n}^{m}$ и получим

$$
\begin{gathered}
f_{e 0 n}^{\text {in }}=-2 i^{n} \sqrt{\frac{n(n+1)}{2 n+1}} \bar{P}_{n}^{1}(\cos \alpha)=h_{n} a_{n}^{\text {in }}, \\
M_{e 0 n, \varphi}^{\mathrm{r}}=\sqrt{\frac{2 n+1}{\pi n(n+1)} j_{n}(k r) \bar{P}_{n}^{1}(\cos \theta)=\frac{1}{h_{n}} \Psi_{e 1 n}^{(1)} \frac{1}{\cos \varphi},}
\end{gathered}
$$

где $h_{n}=\sqrt{n(n+1) /(2 n+1)}$.

Сравним разложения $p$ по формуле (13) с коэффициентами (29) и функциями (30) и разложения $E_{\mathrm{AS}, \varphi} \cos \varphi$ по формулам $(31),(35),(36)$. Очевидно, что для осесимметричной части падающей плоской волны эти разложения почленно совпадают, поскольку множитель $h_{n}$ входит в числитель и знаменатель. Как легко понять, сходное почленное совпадение должно иметь место и для разложения рассеянного поля. Тогда переход между $T$-матрицами, получаемыми для потенциала $p\left(T_{p}^{\mathrm{s}}\right)$ и потенциала Дебая $V_{\mathrm{m}}\left(T_{V}^{\mathrm{s}}\right)$, записывается как

$$
T_{V}^{\mathrm{s}}=H^{-1} T_{p}^{\mathrm{s}} H
$$

где $H=\left\{h_{n} \delta_{l n}\right\}_{l, n=1}^{\infty}-$ диагональная матрица. Аналогично выглядит связь $T$-матриц для потенциалов $q$ и

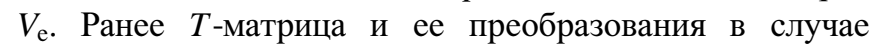
применения потенциалов $p, q$ не рассматривались.

\section{3. Переход от неортогонального базиса к ортогональному}

При нахождении неосесимметричной части полей в сфероидальных координатах предпочтительно использовать базис $\mathbf{M}_{\sigma m n}^{\mathbf{z}}, \mathbf{M}_{\sigma m n}^{\mathbf{r}}$, связанный с потенциалами $U, V$. Соотношение между сферическими $T$-матрицами, порождаемыми данным неортогональным базисом и стандартным ортогональным базисом $\mathbf{M}_{\sigma m n}^{\mathbf{r}}, \mathbf{N}_{\sigma m n}^{\mathbf{r}}$, связанным с потенциалами Дебая, ранее не рассматривалось. Исследуем способ получения такого соотношения в частном случае осесимметричной задачи.

Начнем с разложения ТЕ-моды плоской волны. В случае применения потенциалов $U, V[14]$ имеем

$$
\mathbf{E}^{\mathrm{in}}=\sum_{m=0}^{\infty} \sum_{n=m}^{\infty} a_{e m n}^{\mathrm{in}} \mathbf{M}_{e m n}^{\mathbf{z}},
$$


а при использовании потенциалов Дебая $V_{e}, V_{m}-$

$$
\mathbf{E}^{\mathrm{in}}=\sum_{m=0}^{\infty} \sum_{n=m}^{\infty}\left(f_{e m n}^{\mathrm{in}} \mathbf{M}_{e m n}^{\mathbf{r}}+g_{\text {omn }}^{\mathrm{in}} \mathbf{N}_{\text {omn }}^{\mathbf{r}}\right) .
$$

Для осесимметричной части такой волны $(m=0$ и т.д. - см. подробнее разд. 1.4) получаем из уравнений (38), (39) соотношение

$$
\mathbf{E}_{\mathrm{AS}}^{\mathrm{in}}=\sum_{n=0}^{\infty} a_{e 0 n}^{\mathrm{in}} \mathbf{M}_{e 0 n}^{\mathbf{z}}=\sum_{n=0}^{\infty} f_{e 0 n}^{\mathrm{in}} \mathbf{M}_{e 0 n}^{\mathbf{r}},
$$

где коэффициенты равны [14,32]

$$
\begin{gathered}
a_{e 0 n}^{\text {in }}=i^{n-1} \frac{2(2 n+1)}{\sin \alpha} P_{n}(\cos \alpha), \\
f_{e 0 n}^{\text {in }}=i^{n} \frac{2 n+1}{n(n+1)} \sin \alpha \frac{\mathrm{d} P_{n}(\cos \alpha)}{\mathrm{d} \cos \alpha} .
\end{gathered}
$$

Соотношение (40) с коэффициентами (41) справедливо для любых значений $\alpha$. Проинтегрируем по этому углу от 0 до $\pi$, домножив предварительно на $\sin \alpha P_{n}(\cos \alpha)$, и получим

$$
\mathbf{M}_{e 0 n}^{\mathbf{z}}=\frac{1}{2 n+1}\left(\mathbf{M}_{e 0(n+1)}^{\mathbf{r}}+\mathbf{M}_{e 0(n-1)}^{\mathbf{r}}\right) .
$$

Легко найти, что используемые при рассмотрении ТМмоды функции $\mathbf{N}_{e 0 n}^{\mathbf{z}}$ аналогичным образом выражаются через $\mathbf{N}_{e 0(n+1)}^{\mathbf{r}}$ и $\mathbf{N}_{e 0(n-1)}^{\mathbf{r}}$.

При использовании номированных функций Лежандра имеем

$$
\begin{aligned}
\mathbf{M}_{e 0 n}^{\mathbf{z}}= & \frac{1}{\sqrt{(2 n+1)(2 n+3)}} \mathbf{M}_{e 0(n+1)}^{\mathbf{r}} \\
& +\frac{1}{\sqrt{(2 n-1)(2 n+1)}} \mathbf{M}_{e 0(n-1)}^{\mathbf{r}}
\end{aligned}
$$

или в матричной форме

$$
\mathbf{M}^{\mathbf{z}}=F^{T} \mathbf{M}^{\mathbf{r}},
$$

где $\quad \mathbf{M}^{\mathbf{z}}=\left\{\mathbf{M}_{e 0 n}^{\mathbf{z}}\right\}_{n=1}^{\infty}, \quad \mathbf{M}^{\mathbf{r}}=\left\{\mathbf{M}_{e 0 n}^{\mathbf{r}}\right\}_{n=1}^{\infty} \quad$ и $\quad$ элементы симметричной двухдиагональной матрицы $F$ равны $F_{l n}=\left(\delta_{l, n+1}+\delta_{l, n-1}\right) / \sqrt{(2 n+1)(2 l+1)}$.

Теперь рассмотрим ТЕ-моду рассеянного излучения. Сравним разложения поля при использовании потенциалов Дебая и потенциалов $U, V$ и во втором случае учтем соотношение (44)

$$
\begin{aligned}
\mathbf{E}_{\mathrm{AS}}^{\mathrm{sca}} & =\sum_{n=1}^{\infty} f_{e 0 n}^{\mathrm{sca}} \mathbf{M}_{e 0 n}^{\mathbf{r}}=\sum_{n=1}^{\infty}\left(a_{e 0 n}^{\mathrm{sca}} \mathbf{M}_{e 0 n}^{\mathbf{z}}+b_{e 0 n}^{\mathrm{sca}} \mathbf{M}_{e 0 n}^{\mathbf{r}}\right) \\
& =\sum_{n=1}^{\infty}\left[a_{e 0 n}^{\mathrm{sca}}\left(\sum_{l=1}^{\infty} F_{l n} \mathbf{M}_{e 0 l}^{\mathbf{r}}\right)+b_{e 0 n}^{\mathrm{sca}} \mathbf{M}_{e 0 n}^{\mathbf{r}}\right] \\
& =\sum_{l=1}^{\infty}\left[\sum_{n=1}^{\infty}\left(F_{l n} a_{e 0 n}^{\mathrm{sca}}+b_{e 0 n}^{\mathrm{sca}}\right)\right] \mathbf{M}_{e 0 l}^{\mathrm{r}} .
\end{aligned}
$$

Из уравнений (40) и (45) получаем с учетом соотношения (44) в матричном виде

$$
\mathbf{f}^{\text {in }}=F \mathbf{a}^{\text {in }}, \mathbf{f}^{\text {sca }}=F \mathbf{a}^{\text {sca }}+\mathbf{b}^{\text {sca }} .
$$

При применении потенциалов $U, V$ в сферической системе имеем следующие соотношения $\left(\mathbf{b}^{\text {in }}=0\right)$ :

$$
\mathbf{a}^{\mathrm{sca}}=T_{U V, 11}^{\mathrm{s}} \mathbf{a}^{\mathrm{in}}, \mathbf{b}^{\mathrm{sca}}=T_{U V, 21}^{\mathrm{s}} \mathbf{a}^{\text {in }},
$$

где $T_{U V, i j}^{\mathrm{s}}-4$ блока полной $T$-матрицы $(i, j=1,2)$.

Наконец, из уравнений (46) и (47) получаем соотношение между $T$-матрицами, связанными с неортогональным $\left(T_{U V}^{\mathrm{s}}\right)$ и ортогональным $\left(T_{V V}^{\mathrm{s}}\right)$ базисами:

$$
T_{V V}^{\mathrm{s}}=\left(F T_{U V, 11}^{\mathrm{s}}+T_{U V, 21}^{\mathrm{s}}\right) F^{-1} .
$$

Таким же образом, т. е. используя разложение плоской волны, можно получить связь функций $\mathbf{M}_{\sigma m n}^{\mathrm{z}}$ и $\mathbf{M}_{\sigma m n}^{\mathrm{r}}$ в общем случае $m \neq 0$ и далее найти сходное с формулой (48) соотношение между $T$-матрицами, связанными с неортогональным и ортогональным базисами, для неосесимметричной части полей.

Отметим, что преобразование $T$-матриц при переходе от неортогонального к ортогональному сферическому базису и обратно ранее никогда не исследовалось.

\section{3. Результаты численных расчетов и их обсуждение}

Основными результатами работы являются формулы (28), (37), (48), которые базируются на соотношениях (24), (25), (42). Первые два из них были известны ранее, но их не удалось в полной мере протестировать для сфероидальных функций 3-го рода из-за их не совсем аккуратного вычисления в оригинальной статье [35], третье соотношние является новым. Поэтому прежде всего в настоящей работе мы убедились, что все эти три соотношения верны, проведя численные расчеты с высокой точностью как для азимутального числа $m=0$, так и для $m>0$.

Соотношение (28), первое из найденных нами, связывает $T$-матрицы, полученные для сфероидального и сферического базисов, порожденных одинаковыми потенциалами. Для набора параметров мы рассчитали сфероидальные матрицы $T_{p, q}^{\mathrm{sp}}$ для потенциалов $p, q$ по формулам из разд. 1.4. Затем, используя уравнение (28), вычислили сферические матрицы $T_{p, q}^{\mathrm{s}}$ и для падающей плоской волны по этим $T$-матрицам нашли коэффициенты разложения рассеянного поля $a_{m n}^{\text {sca }}, b_{m n}^{\text {sca }}$ и далее сечения ослабления $C_{\text {ext }}$ и рассеяния $C_{\text {sca. }}$. При этом для сфероидальных координат использовались соотношения (23), а для сферических - аналогичные выражения с заменой $\bar{S}_{1 l}\left(c_{1}, \cos \alpha\right)$ на $\bar{P}_{l}^{1}(\cos \alpha)$. Такие же вычисления были проведены для потенциалов $U, V$ при $m=0$ (матрицы $T_{U, V}^{\mathrm{sp}}$ и т.д.). Рассмотрение сечений вместо $T$-матриц является более компактным и фокусирует внимание на элементах матриц, наиболее важных для определения 

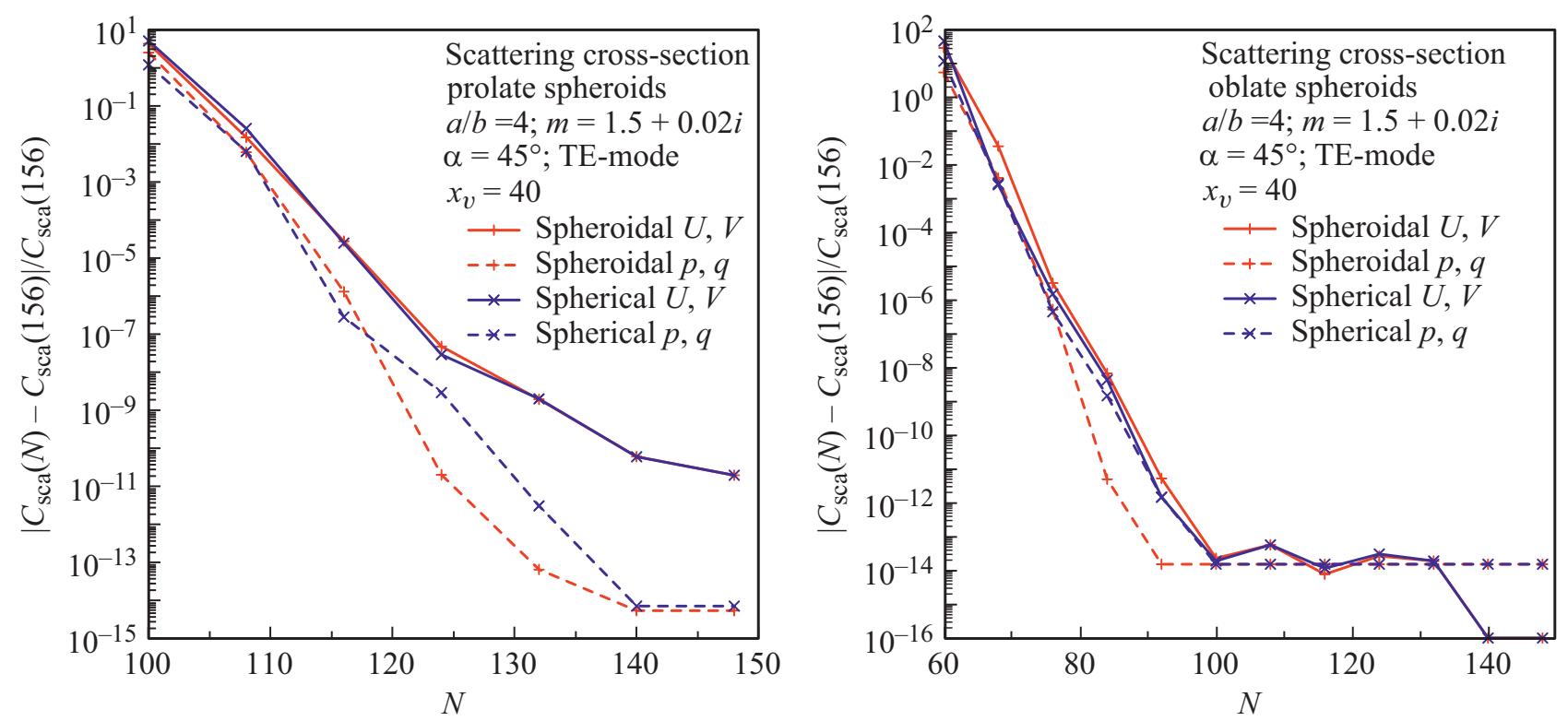

Рис. 1. Сходимость сечений рассеяния $C_{\text {sса }}(N)$ (к значению при $N=156$ ) с ростом числа $N$ слагаемых, учитываемых в разложениях для вытянутых (левая панель) и сплюснутых (правая панель) сфероидов в осесимметричной задаче.
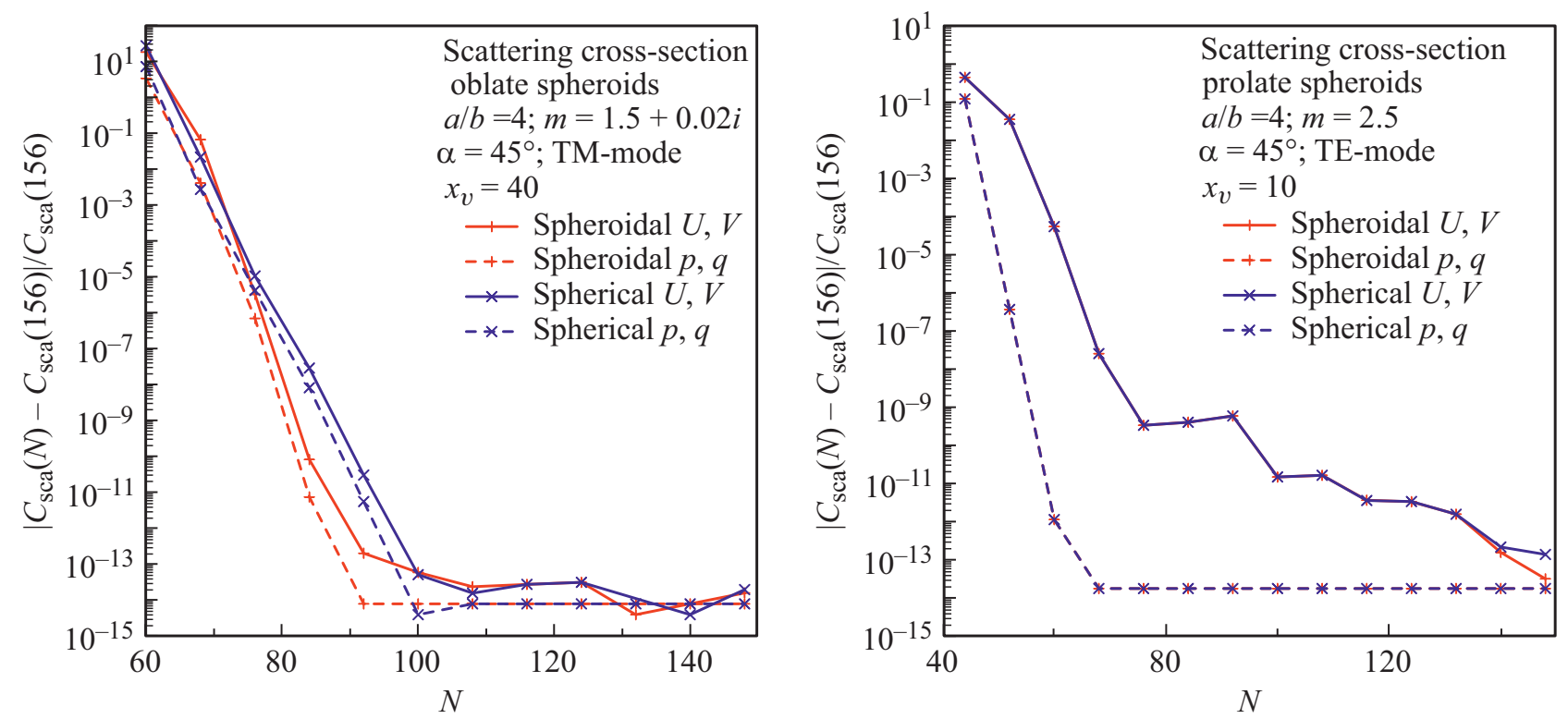

Рис. 2. То же, что на рис. 1, но для ТМ-моды (левая панель) и показателя преломления $m=2.5$ (правая панель).

полей в дальней зоне, в которой в большинстве случаев применяются программы светорассеяния.

На рис. 1,2 приведены некоторые результаты наших вычислений, а именно сечения рассеяния, полученные при расчетах с двойной точностью для вытянутых и сплюснутых сфероидов с отношением полуосей $a / b=4$, показателем преломления $m=1.5+0.02 i$ и дифракционным параметром $x_{\mathrm{v}}=40$, а также с $m=2.5$ и $x_{\mathrm{v}}=10$; плоская волна падала на частицы под углом к их оси симметрии $\alpha=45^{\circ}$. Рисунок 1 иллюстрирует результаты преобразования „сфероидальная $\rightarrow$ сферическая“ для $T$ матриц, основанных на использовании вытянутых (левая панель) и сплюснутых (правая панель) сфероидальных функций. На рис. 2 показаны результаты этого преобразования для другой моды и вещественного показателя преломления.

Важнейший вывод, который позволяют сделать приведенные рисунки, состоит в том, что преобразование (28) дает возможность вычислить сферическую $T$-матрицу в области значений параметров задачи, в которой обычный метод $T$-матриц сделать этого не позволяет. В частности, наиболее продвинутый алгоритм этого метода SMARTIES дает сходящееся решение для частиц с $a / b=4$ и $m=1.5+0.02 i$ только при $x_{\mathrm{v}}<16$ для 
Факторы эффективности $Q_{\text {sca }}^{\text {AS }}$ рассчитанные по разным $T$-матрицам для вытянутых и сплюснутых сфероидов с параметрами $a / b=4, x_{\mathrm{v}}=40, m=1.5+0.02 i, \alpha=45^{\circ}$, ТЕ-мода осесимметричной задачи

\begin{tabular}{l|c|c|c}
\hline Координаты & Потенциалы & Вытянутые & Сплюснутые \\
\hline Сфероидальные & $p, q$ & 0.0516640899039573 & 0.0261366229316886 \\
Сферические & $p, q$ & 0.0516640899039574 & 0.0261366229316886 \\
Сфероидальные & $U, V$ & 0.0516640900725206 & 0.0261366229316860 \\
Сферические & $U, V$ & 0.0516640900725207 & 0.0261366229316860
\end{tabular}

сплюснутых сфероидов и $x_{\mathrm{v}}<12$ для вытянутых [37]. Наша сфероидальная $T$-матрица и соотношение (28) дали весьма точную сферическую $T$-матрицу далеко за пределами этой области. Добавим, что мы проводили вычисления в области значений параметров, в которой ни один подход не дает сферической $T$-матрицы: стандартное использование четверной точности позволяет достигать лишь $x_{\mathrm{v}} \approx 20$ [38], а более универсальные подходы ограничены (при данных значениях параметров, но при использовании существенно больших ресурсов) областью $x_{\mathrm{v}}<10-40$ (см., например, метод дискретных диполей в [39]).

Рассмотрим более детально результаты расчетов перехода от сфероидальной $T$-матрицы к сферической. Заметим, что если для потенциалов $p, q$ сфероидальная матрица $T$ считается хорошо, то, как можно судить по результатам, для потенциалов $U, V$ (при $m=0)$ в случае вытянутых сфероидов сфероидальная $T$-матрица рассчитывается не совсем точно. Во всех рассмотренных нами случаях с ростом числа учитываемых слагаемых $N$ (размер $T$-матрицы) сечения, рассчитанные по сфероидальной и сферической $T$-матрицам, сходятся, причем к одному и тому же значению (таблица). Для вещественного показателя преломления подобное согласие сфероидальной и сферической $T$-матриц всегда получалось очень хорошим (правая панель рис. 2). Также согласие лучше для матриц, найденных для потенциалов $p, q$ по сравнению с $U, V$.

Обращает на себя внимание то, что при одном и том же размере $T$-матриц $N$ сечения рассеяния, рассчитанные для поглощающих сфероидов по найденной сферической матрице, могут быть на несколько значащих цифр менее точными, чем рассчитанные по сфероидальной матрице (левые панели рис. 1 и 2). Это происходит, когда добавляемые члены в разложение для сечения дают вклад, меньший примерно $10^{-6}$ от точного значения. Эффект, по-видимому, связан с разными последствиями ошибок округления. Заметим, что данный дефект полностью компенсируется тем, что с ростом $N$ он всегда нивелируется.

Второе найденное в работе соотношение (37) было проверено сравнением матрицы $T_{p, q}^{\mathrm{s}}$, полученной с использованием соотношения (28) при $m=1$ из матрицы $T_{p, q}^{\mathrm{sp}}$, рассчитанной по формулам разд. 1.4, с матрицей $T_{V V}^{\mathrm{s}}$, выдаваемой программой из монографии [32], основанной на разложении полей по сферическому ба- зису, связанному с потенциалами Дебая, и также на применении метода расширенных граничных условий (разд. 1.1). Во всех рассмотренных случаях мы нашли полное согласие матриц $T_{p, q}^{\mathrm{s}}$ и $T_{V V}^{\mathrm{s}}$ в пределах точности вычислений.

Третье полученное нами соотношение (48) было протестировано сравнением матрицы $T_{U, V}^{\mathrm{s}}$, полученной для осесимметричной задачи $(m=0)$ из соотношения $(28)$, с матрицей $T_{V V}^{\mathrm{s}}$, обсуждавшейся в предыдущем абзаце. Во всех случаях согласие матриц было полным, если учитывать использованную точность вычислений. Единственной особенностью, которую следует отметить, является специфика соотношения (44): оно верно при бесконечном числе рассматриваемых функций, а при конечном - технически не учитывается первое слагаемое двухчленной формулы (43). Однако с ростом числа базисных функций этот эффект становится малосущественным.

\section{Заключение}

На основе решения осесимметричной задачи рассеяния света сфероидом в сфероидальной системе координат, связанной с частицей, найдено и проверено соотношение между $T$-матрицами, получаемыми при разложении полей в сфероидальном и сферическом базисах, связанных с одинаковыми скалярными потенциалами.

Предложен подход к построению соотношения между $T$-матрицами, возникающими при использовании неортогонального (но эффективно применяемого в методе разделения переменных в сфероидальных координатах) и стандартного ортогонального сферического базисов.

Выполненные численные расчеты показали высокую точность найденных соотношений между $T$-матрицами. Отмечено, что вычисление сфероидальной $T$-матрицы и ее преобразование с помощью полученных формул в стандартную сферическую $T$-матрицу, используемую в приложениях для расчета оптических свойств хаотически ориентированных частиц, является единственным способом рассчитать последнюю в широкой области значений дифракционного параметра и отношения полуосей сфероида. 


\section{Финансирование работы}

Работа В.Ф. по теоретическому изучению $T$-матриц была поддержана Министерством науки и высшего образования РФ, соглашение № FSRF-2020-0004, работа В.И. и Д.Т. по расчетам Т-матриц - грантом РНФ 2072-10052.

\section{Конфликт интересов}

Авторы заявляют, что у них нет конфликта интересов.

\section{Список литературы}

[1] O. Dubovik, A. Sinyuk, T. Lapyonok, B.N. Holben, M.I. Mishchenko, P. Yang, T.F. Eck, H. Volten, O. Munoz, B. Veihelmann. J. Geophys. Res. Atmos., 111, D11208 (2006). DOI: 10.1029/2005JD006619

[2] S. Merikallio, H. Lindqvist, T. Nousiainen, M. Kahnert. Atmos. Chem. Phys., 11 (11), 5347 (2011). DOI: 10.5194/acp-115347-2011

[3] H. Tang, X.-X. Li. Int. J. Num. Meth. Heat Fluid Flow, 24 (8), 1762 (2014). DOI: 10.1108/HFF-04-2013-0105

[4] N.V. Voshchinnikov, V.B. Il'in, H.K. Das. Mon. Not. Roy. Astron. Soc., 462 (3), 2343 (2016). DOI: $10.1093 / \mathrm{mnras} / \mathrm{stw} 1751$

[5] Thermo Fisher Scientific [Электронный ресypc]. URL: https://www.thermofisher.com/ru/ru/home/life-science/cellculture/organoids-spheroids-3d-cell-culture.html

[6] A. Sihvola. J. Nanomater., 2007, 045090 (2007). DOI: $10.1155 / 2007 / 74545$

[7] R. Hogg. KONA Powder Part. J., 32, 227 (2015). DOI: $10.14356 /$ kona.2015014

[8] B.T. Draine, B.S. Hensley. Astrophys. J., 910 (1), 47 (2021). DOI: $10.3847 / 1538-4357 / a b d d b 7$

[9] B. Vandenbroucke, M. Baes, P. Camps, A.U. Kapoor, D. Barrientos, J.-P. Bernard. Astron. Astrophys., 653 (1), A34 (2021). DOI: 10.1051/0004-6361/202141333

[10] H. Chen-Chen, S. Pérez-Hoyos, A. Sánchez-Lavega. Icarus., 354 (11), 114021 (2021). DOI: 10.1016/j.icarus.2020.114021

[11] B. Jaiswal, G. Mahapatra, A. Nandi, M. Sudhakar, K. Sankarasubramanian, V. Sheel. Planet. Spa. Sci., 201 (10), 105193 (2021). DOI: 10.1016/j.pss.2021.105193

[12] S. Höfer, H. Mutschke, Th.G. Mayerhöfer. Astron. Astrophys., 646 (2), A87 (2021). DOI: 10.1051/0004-6361/202038931

[13] I.R. Ciric, F.R. Cooray. In: Light scattering by nonspherical particles, ed. by M.I. Mishchenko, J.W. Hovenier, L.D. Travis (Academic Press, San Diego, 2000), p. 89.

[14] N.V. Voshchinnikov, V.G. Farafonov. Astrophys. \& Space Sci., 204 (1), 19 (1993). DOI: 10.1007/BF00658095

[15] M.I. Mishchenko, L.D. Travis, A. Lacis. Scattering, absorption and emission of light by small particles (Cambridge Univ. Press, Cambridge, 2002).

[16] H.K. Das, N.V. Voshchinnikov, V.B. Il'in. Mon. Not. Roy. Astron. Soc., 404 (1), 265 (2010). DOI: 10.1111/j.13652966.2010.16281.x

[17] M. Min. EPJ Web Conf., 102 (1), 00005 (2015). DOI: $10.1051 /$ epjconf/201510200005

[18] A.K. Ospanova, A. Basharin, A.E. Miroshnichenko, B. Luk'yanchuk. Opt. Mater. Expr., 11 (1), 23 (2021). DOI: 10.1364/OME.414340
[19] M.I. Mishchenko, J.W. Hovenier, L.D. Travis. Light scattering by nonspherical particles (Academic Press, San Diego, 2000).

[20] F.M. Kahnert. J. Quant. Spectrosc. Rad. Transf., 79-80 (1), 775 (2003). DOI: 10.1016/S0022-4073(02)00321-7

[21] S. Asano, G. Yamamoto. Appl. Opt., 14 (1), 29 (1975). DOI: $10.1364 / A O .14 .000029$

[22] L.-W. Li, X.-K. Kang, M.-S. Leong. Spheroidal wave functions in electromagnetic theory (John Wiley \& Sons, New York, 2002).

[23] А.А. Абрамов, Е.Д. Калинина, С.В. Курочкин. Ж. вычисл. матем. и матем. физ., $55(5), 798$ (2015). DOI: $10.7868 / \mathrm{S} 0044466915050038$ [A.A. Abramov, E.D. Kalinin, S.V. Kurochkin. Comput. Math. Math. Phys., 55, 788 (2015). DOI: 10.1134/S0965542515050036].

[24] L.A. van Buren. Препринт arXiv.org (math) [Электронный pecypc]. URL: https://arxiv.org/abs/2009.01618

[25] M.I. Mishchenko. J. Quant. Spectrosc. Rad. Transf., 242, 106692 (2020). DOI: 10.1016/j.jqsrt.2019.106692

[26] К. Борен, Д. Хафмен. Поглощение и рассеяние света малыми частицами (Мир, М., 1986). [C. Bohren, D. Huffman. Absorption and scattering of light by small particles (J. Wiley \& Sons, New York, 1983)].

[27] V.G. Farafonov, V.B. Il'in. Light Scatt. Rev., 1, 125 (2006).

[28] V.G. Farafonov. Light Scatt. Rev., 8, 189 (2013). DOI: https://doi.org/10.1007/978-3-642-32106-1_5

[29] В.И. Комаров, Л.И. Пономарев, С.Ю. Славянов. Сбероидальные и кулоновские сфероидальные бункции (Наука, M., 1976).

[30] C. Flammer. Spheroidal wave functions (Stanford Univ. Press, 1957).

[31] G. Mie. Ann. Phys., 330 (25), 377 (1908). DOI: $10.1002 /$ andp. 19083300302

[32] P.W. Barber, S.C. Hill. Light scattering by particles: computational methods (World Scientific, Singaphore, 1990). DOI: $10.1142 / 0784$

[33] В.Г. Фарафонов. Дифференц. уравн., 19 (10), 1765 (1983).

[34] V.G. Farafonov, N.V. Voshchinnikov. Appl. Opt., 51 (10), 1586 (2012). DOI: 10.1364/AO.51.001586

[35] В.Г. Фарафонов, Н.В. Вощинников, Е.Г. Семенова. Зап. науч. сем. ПОМИ, 426, 203 (2014). [V.G. Farafonov, N.V. Voshchinnikov, E.G. Semenova. J. Math. Sci., 214 (3), 382 (2016). DOI: 10.1007/s10958-016-2784-3].

[36] M.F. Schulz, K. Stamnes, J. Stamnes. Appl. Opt., 37 (33), 7875 (1998). DOI: 10.1364/AO.37.007875

[37] W.R.C. Somerville, B. Auguiè, E.C. Le Ru. J. Quant. Spectrosc. Rad. Transf., 174 (1), 39 (2016). DOI: $10.1016 /$ j.jqsrt.2016.01.005

[38] M.I. Mishchenko, L.D. Travis. Opt. Commun., 109 (5), 16 (1994). DOI: 10.1016/0022-4073(96)00002-7

[39] M.A. Yurkin, A.G. Hoekstra. J. Quant. Spectrosc. Rad. Transf., 112 (13), 2234 (2011). DOI: 10.1016/j.jqsrt.2011.01.031 